\title{
Delayed PCI 12 Hours after the Onset of Symptoms Is Associated with Improved Outcomes for Patients with ST-Segment Elevation Myocardial Infarction: A Real-World Study
}

\author{
Wen-Juan Xiu, Hai-Tao Yang, Ying-Ying Zheng $\mathbb{D}$, Yi-Tong Ma, and Xiang Xie $\mathbb{1}$ \\ Heart Center, First Affiliated Hospital of Xinjiang Medical University, Urumqi, Xinjiang 830011, China \\ Correspondence should be addressed to Xiang Xie; xiangxie999@sina.com
}

Received 30 November 2018; Revised 20 April 2019; Accepted 6 May 2019; Published 18 June 2019

Academic Editor: Andrea Rubboli

Copyright (C) 2019 Wen-Juan Xiu et al. This is an open access article distributed under the Creative Commons Attribution License, which permits unrestricted use, distribution, and reproduction in any medium, provided the original work is properly cited.

\begin{abstract}
Background. Primary percutaneous coronary intervention (PPCI) plays a pivotal role in the treatment of ST-segment elevation myocardial infarction (STEMI). However, it remains controversial whether PCI delayed beyond the recommended time window of $12 \mathrm{~h}$ after the onset of symptoms is applicable to STEMI. Objective. The acute myocardial infarction (AMI) registration study in Xinjiang, China, is a real-world clinical trial (retrospective cohort study) that includes hospitalized patients. The purpose of this study was to compare delayed PCI and medication therapy beyond the recommended time window of $12 \mathrm{~h}$ after the onset of symptoms on the outcomes of STEMI patients. Methods and Results. From May 2012 to December 2015, a total of 1072 STEMI patients received delayed PCI $(n=594)$ or standard medication therapy (MT) $(n=478)$ more than $12 \mathrm{~h}$ after the onset of symptoms. The number of all-cause deaths in the delayed PCI group and that in the MT group were 55 (9.3\%) and 138 (28.9\%), respectively, and a significant difference between the groups was indicated for this variable $(\mathrm{P}<0.001)$. The number of cardiac deaths in the delayed PCI group and that in the medication therapy group were $47(7.9 \%)$ and $120(25.1 \%)$, respectively, and a significant difference between the groups was indicated for this variable $(\mathrm{P}<0.001)$. We also found that the MACE incidence in the delayed PCI group was significantly higher than it was in the MT group (32.2\% versus $43.5 \%, \mathrm{P}<0.001)$. Propensity score matching (PSM) analyses remained significant differences between the delayed PCI group and the MT group, respectively, in all-cause deaths $(9.3 \%$ versus $25.8 \%, \mathrm{P}<0.001)$ and cardiac death $(8.7 \%$ versus $21.6 \%, \mathrm{P}<0.001)$. Conclusion. Compared to medication therapy, PCI for STEMI delayed beyond $12 \mathrm{~h}$ after the onset of symptoms can better reduce mortality and the incidence of MACEs. Trial Registration. This study is registered with the following: Trial Registration: clinicaltrials.gov; Identifier: NCT02737956.
\end{abstract}

\section{Introduction}

Primary percutaneous coronary intervention (PPCI) is an important treatment for ST-segment elevation myocardial infarction (STEMI) [1, 2]. Early myocardial reperfusion is the main target of treatment for acute myocardial infarction (AMI). Existing guidelines have already emphasized the importance of reperfusion of the infarct-related artery (IRA) in this treatment [3]. After AMI, salvaging the myocardium at the earliest possible time is critical. PPCI can open up the IRA, reducing the infarct area and residual stenosis, preserving and improving left ventricular function, and preventing reocclusion. Reducing the time delay of salvaging the myocardium is the primary concern in reperfusion therapy for STEMI. The time from the first medical contact (FMC) to PCI should be reduced, preferably to less than 90 min (I, A). The time from FMC to discharge should also be reduced to lower the risk of in-hospital mortality. ACC/AHA STEMI guidelines recommend performing PPCI within $90 \mathrm{~min}$ of FMC (class 1 recommendation) and performing treatment within $120 \mathrm{~min}$ of complete ischemia [4, 5]. The European Society of Cardiology (ESC) guidelines also make a similar recommendation for $\mathrm{AMI}$, which is to shorten the time from the onset of STEMI to PCI $[6,7]$. In clinical practice, PCI is usually performed within $12 \mathrm{~h}$ following STEMI. However, in real-world situations, many patients go to the 
hospital beyond the $12 \mathrm{~h}$ window of the onset of chest pain. Thus, it is disputed whether delayed PCI can deliver the desired outcome. Some studies have shown that standard or optimized medication therapy is better than delayed PCI $[8,9]$, while others argue for the benefits of delayed PCI for remedy reperfusion therapy [10-16]. The accepted timing of PCI ranges from 2 days to 60 days after the onset of STEMI, though the long-term prognosis of each differs and some contradictory conclusions have been drawn $[17,18]$.

The 2016 Chinese guidelines for PCI consider performing PCI on those with clinical ischemia and (or) evidence of ischemia within 12-48 $\mathrm{h}$ after the onset of symptoms to be feasible (class of recommendation IIa, level of evidence B) [19]. However, there is a lack of evidence of whether PPCI should be performed beyond $12 \mathrm{~h}$ after the onset symptoms in those without explicit evidence of ischemia. This study compared the clinical outcomes of STEMI patients who received delayed PCI and STEMI patients who received medication therapy.

\section{Methods}

2.1. Study Design. The registration study of AMI in Xinjiang, China, is a multicenter clinical trial initiated by the First Affiliated Hospital of Xinjiang Medical University without any sponsorship from enterprises. Consecutive patients with STEMI who were hospitalized at the First Affiliated Hospital of Xinjiang Medical University from May 2012 to December 2015 were recruited. The research protocol was approved by the ethics committee or review committee of the First Affiliated Hospital of Xinjiang Medical University. Because the study was a retrospective cohort study based on realworld situations, there was no need to obtain informed consent from the patients.

2.2. Subjects. A total of 1072 STEMI patients who received treatment beyond the $12 \mathrm{~h}$ time window at the First Affiliated Hospital of Xinjiang Medical University were included in this study. The inclusion criteria were as follows: (1) Continuous chest pain for over $30 \mathrm{~min}$ without remission; (2) ST-elevation on ECG (without left ventricular hypertrophy or a left bundle branch block): J-point elevation in two adjacent leads, the cutoff being $\geq 0.2 \mathrm{mV}$ for men and $\geq 0.15 \mathrm{mV}$ for women, and (or) a cutoff of $\geq 0.1 \mathrm{mV}$ in the other leads; (3) appearance of a pathological Q-wave on ECG; (4) New radiologic finding of a loss of viable myocardium or local heart wall motion abnormality; (5) beyond the $12 \mathrm{~h}$ window after the onset of chest pain. The patients were excluded if they met one or more of the following criteria: (1) tumor, liver, and kidney insufficiency; (2) life expectancy less than 6 months; (3) $\mathrm{LVEF}<30 \%$; (4) previous thrombolysis recipient.

2.3. PCI Group. The patients were given oxygen therapy, analgesics, and standard medication therapy. The medication therapy consisted of $75 \mathrm{mg}$ oral clopidogrel once daily, $100 \mathrm{mg}$ aspirin once daily, and low-molecular-weight heparin and/or glycoprotein IIb/IIIa antagonists. The patients were given the preoperative load (150 mg clopidogrel) on the night before surgery. All STEMI patients without contraindications were given statins, $\beta$-receptor inhibitors, ACEI, or ARB as preventive therapy. The patients received coronary angiography (CAG) to determine the necessity of stent implantation in IRA. Non-IRA was also treated appropriately. Occlusion of IRA was defined as $100 \%$ stenosis with a TIMI grade of 0 or 1 .

2.4. Medication Therapy Group. The patients were given oxygen therapy, analgesics, and standard medication. The standard medication was the same as that used in the PCI group.

2.5. Clinical Endpoints and Follow-Up. The primary endpoints were all-cause death; the secondary endpoint was MACE, which consisted of cardiac death, revascularization, reinfarction, stroke, and rehospitalization for heart failure. We also recorded bleeding events. All patients were followed up by telephone or mail. MACEs were observed, and every endpoint event was recorded. A single patient might undergo one or several MACEs, but all of them were recorded. The median follow-up was 26 months (0-55 months).

2.6. Data Analysis. The baseline data and continuous variables of CAG that showed a normal distribution were presented as the mean \pm standard deviation (SD). The measurement data were analyzed by an independent samples t-test, and the count data were analyzed by the chi-square test. Kaplan-Meier survival curves were drawn for univariate survival analysis. The survival rates between the subgroups were examined by the log-rank test. Multivariate survival analysis was conducted using the Cox regression model.

To reduce selection bias and potential confounding factors, propensity score matching (PSM) was adopted for the adjustment of baseline clinical characteristics. After 1:1 matching, 666 patients were selected for the final analysis, including 333 AMI patients who received delayed PCI and 333 AMI patients who received medication therapy. The following variables were considered: age, gender, heart rate, smoking status, and Killip grade. The chi-square test and independent samples t-test were reimplemented during matching to test for the differences in the variables between the two groups after matching. All data were analyzed statistically using SPSS 24.0. $\mathrm{P}<0.05$ was considered significant.

\section{Results}

A total of 1072 STEMI patients hospitalized from May 2012 to December 2015 were included, with 594 patients in the delayed PCI group $(12 \mathrm{~h}<\mathrm{t}<28 \mathrm{~d})$ and 478 patients in the medication therapy group.

The patients were aged $24-95$ years $(58.49 \pm 12.81$ years), and there were 877 (81.8\%) men and 195 (18.2\%) women. There were 259 patients with diabetes, accounting for $24.2 \%$ of the total patient population; 330 patients with hypertension, accounting for $31 \%$ of the total patient population; and 252 patients with hyperlipidemia, accounting for $23.5 \%$ of the total patient population. According to the Killip classification, there were 23 patients with grade 1 cardiac function, accounting for $2.1 \%$ of the total patient population; 817 patients with grade 2 cardiac function, accounting for 
$76.2 \%$ of the total patient population; and 232 patients with grade 3-4 cardiac function, accounting for $21.7 \%$ of the total patient population.

3.1. Comparison of Baseline Data. Compared with those in the delayed PCI group, the patients in the medication therapy group were older $(\mathrm{P}<0.001)$ and had a higher heart rate $(\mathrm{P}<0.001)$ and worse cardiac function $(\mathrm{P}<0.001)$, and there were more men in this group than there were in the delayed PCI group $(\mathrm{P}=0.004)$. The two groups showed a significant difference in smoking status as a high-risk factor $(\mathrm{P}=0.003)$. However, there was no significant difference in total cholesterol, low-density lipoprotein cholesterol or the prevalence of high-risk factors for coronary heart disease, such as diabetes, hypertension, and hyperlipidemia (all $\mathrm{P}>0.05$ ) (Table 1).

3.2. Primary Endpoints. The number of all-cause deaths in the delayed PCI group and that in the medication therapy group were $55(9.3 \%)$ and $138(28.9 \%)$, respectively, which indicates a significant difference $(\mathrm{P}<0.001)$. The incidence of all-cause death was much higher in the medication therapy group than it was in the delayed PCI group (Table 2).

3.3. All-Cause Death. Kaplan-Meier survival curves and the log-rank test (pooled over strata) revealed a significant difference in the incidence of all-cause death between the two groups $(\mathrm{P}<0.001)$ (Figure $1(\mathrm{a}))$.

Potential influence of factors of survival was analyzed using the multivariate model. After eliminating the interactions between the factors influencing all-cause death, a Cox regression model indicated that the treatment regimen was an independent prognostic factor in STEMI $(\mathrm{P}<0.001)$. Compared with the prognosis in the medication therapy group, the prognosis in the delayed PCI group was considerably improved. The risk of all-cause death was lower after delayed PCI than that after medication therapy $(\mathrm{HR}=0.262,95 \% \mathrm{CI}$ : 0.164-0.417, $\mathrm{P}<0.001$, Table 3).

3.4. Secondary Endpoints. The numbers of patients presenting with MACE were 191 (32.2\%) and 208 (43.5\%) in the delayed PCI group and medication therapy group, respectively, and a significant difference between the groups was shown $(\mathrm{P}<0.001)$. The number of cardiac deaths in the delayed PCI group and that in the medication therapy group were $47(7.9 \%)$ and 120 (25.1\%), respectively, and a significant difference between the groups was shown $(\mathrm{P}<0.001)$. The incidence of MACE and cardiac death was much higher in the medication therapy group than in the delayed PCI group (Table 2).

3.5. MACE. Kaplan-Meier survival curves and the log-rank test (pooled over strata) found a significant difference in the incidence of MACE between the two groups $(\mathrm{P}=0.001)$ (Figure 1(a)).

After eliminating the interaction between the influencing factors for MACE, a Cox regression model found that the treatment regimen was an independent influencing factor for MACE in STEMI patients. Compared with medication therapy, delayed PCI $(12 \mathrm{~h}<\mathrm{t}<28 \mathrm{~d})$ greatly improved the prognosis. The risk of MACE in the patients who received delayed PCI was lower than that in the patients who received medication therapy $(\mathrm{HR}=0.528,95 \% \mathrm{CI}: 0.406$ 0.686, $\mathrm{P}<0.001$, Table 3).

3.6. Cardiac Death. Kaplan-Meier survival curves and the log-rank test (pooled over strata) revealed a significant difference in the incidence of cardiac death between the two groups $(\mathrm{P}<0.001)$ (Figure $1(\mathrm{a})$ ).

Potential influencing factors of survival were analyzed using the multivariate model. After eliminating the interactions between the influencing factors of cardiac death, a Cox regression model indicated that the treatment regimen was an independent prognostic factor in STEMI $(\mathrm{P}<0.001)$. Compared with the prognosis in the medication therapy group, the prognosis in the delayed PCI group was considerably improved. The risk of cardiac death was lower after delayed PCI than after medication therapy $(\mathrm{HR}=0.286,95 \% \mathrm{CI}: 0.173$ 0.474, $\mathrm{P}<0.001$ ) (Table 3).

3.7. PSM Analysis. PSM analysis was used for adjustment of the baseline clinical characteristics. After 1:1 matching, 666 patients were selected for the final analysis, including 333 AMI patients who received delayed PCI and 333 AMI patients who received medication therapy.

The patients were aged $24-94$ years $(58.49 \pm 12.81$ years), and there were 539 (80.9\%) men and 127 (19.1\%) women. There were 168 patients with diabetes, accounting for $25.2 \%$ of the total patient population; 192 patients with hypertension, accounting for $28.2 \%$ of the total patient population; and 153 patients with hyperlipidemia, accounting for $22.9 \%$ of the total patient population. According to Killip classification, there were 13 patients with grade 1 cardiac function, accounting for $2 \%$ of the total patient population; 514 patients with grade 2 cardiac function, accounting for $76.2 \%$ of the total patient population; and 232 patients with grade 3-4 cardiac function, accounting for $20.9 \%$ of the total patient population.

The number of all-cause deaths in the PCI group and that in the medication therapy group were 31 (9.3\%) and 86 $(25.8 \%)$, respectively $(\mathrm{P}<0.001)$. The incidence of MACE was much higher in the medication therapy group than in the delayed PCI group (43.5 versus $38.4 \%, \mathrm{P}=0.092$ ). The number of cardiac deaths in the delayed PCI group and that in the medication therapy group were $29(8.7 \%)$ and $72(21.6 \%)$, respectively $(\mathrm{P}<0.001)$ (Table 4$)$.

Kaplan-Meier survival curves and the log-rank test (pooled over strata) indicated a significant difference in the incidence of all-cause death, MACE, and cardiac deaths between the two groups $(\mathrm{P}<0.001, \mathrm{P}=0.001$, and $\mathrm{P}<0.001$, respectively) (Figure 1(b)). These results indicated that, compared to medication therapy, delayed PCI significantly improved the prognosis of STEMI patients.

\section{Discussion}

Our results indicated that, beyond a $12 \mathrm{~h}$ time window, delayed PCI was superior to standard medication therapy in improving the prognosis of STEMI patients. 


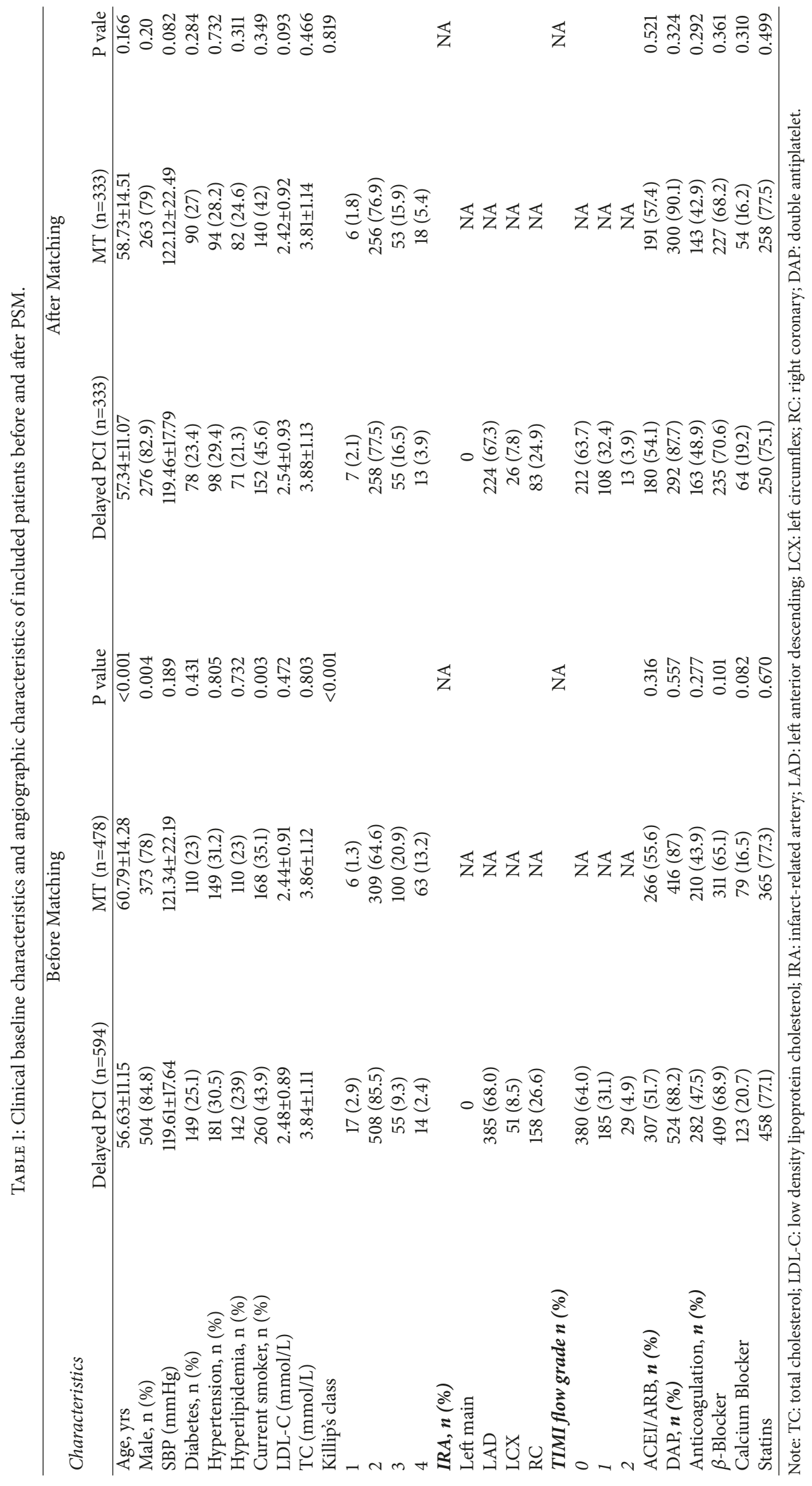




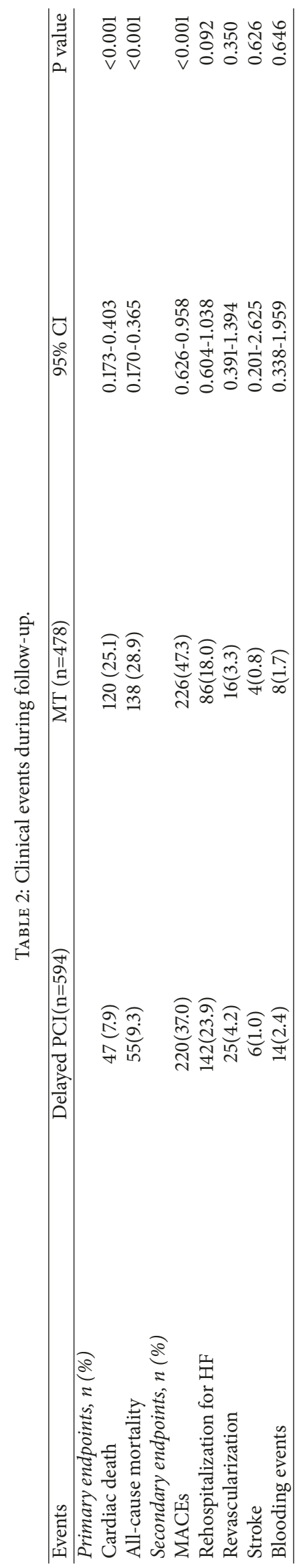


Survival Function for patterns 1 - 2

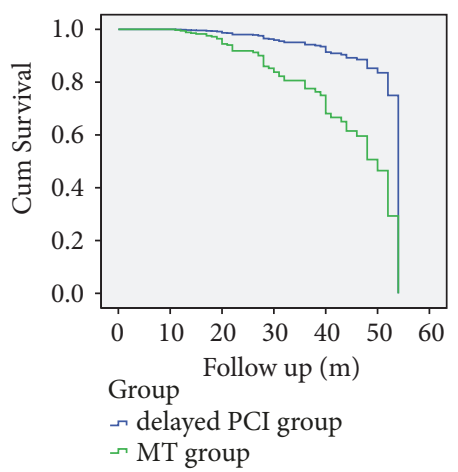

Survival Function for patterns 1 - 2

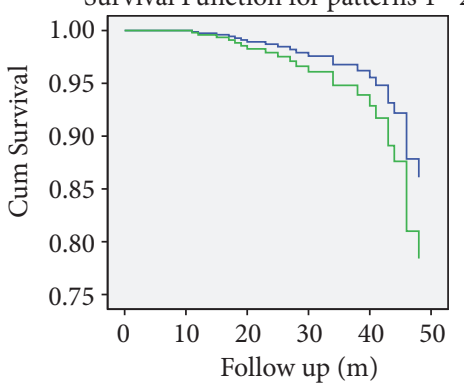

Group

$\neg$ delayed PCI group

$\neg$ MT group

Survival Function for patterns 1 - 2

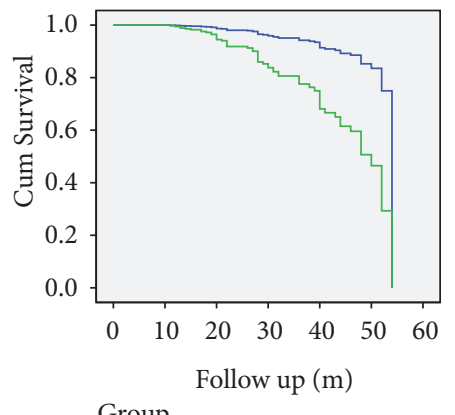

Group

ـ delayed PCI group

$\rightarrow$ MT group

Survival Function for patterns 1 - 2

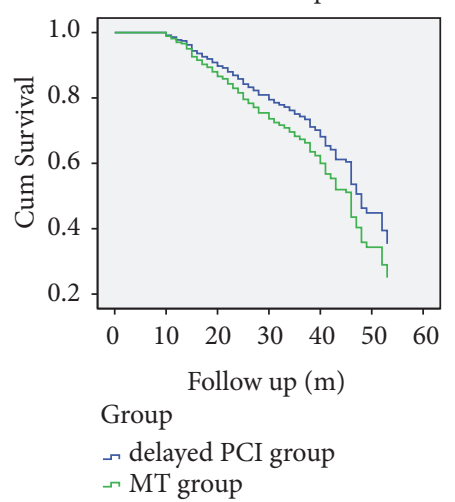

Survival Function for patterns 1 - 2

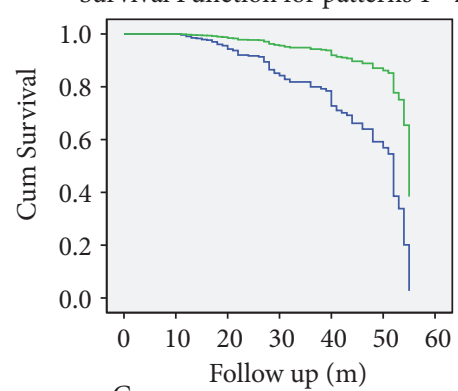

Group

$\rightarrow$ delayed PCI group

$\neg$ MT group

Survival Function for patterns 1 - 2

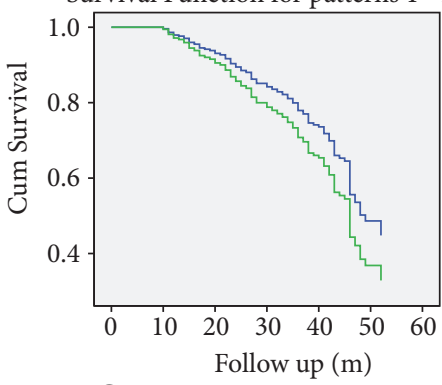

Group

$\neg$ delayed PCI group

$\neg$ MT group

(a)

Survival Function for patterns 1 - 2

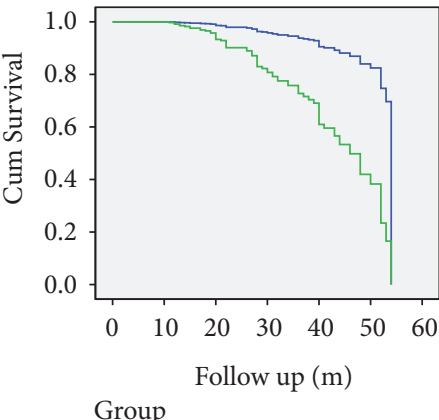

Group

$\neg$ delayed PCI group

$\rightarrow$ MT group

Survival Function for patterns 1 - 2

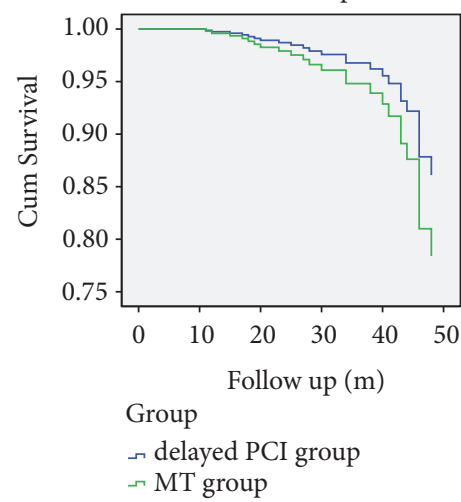

(b)
Survival Function for patterns 1 - 2

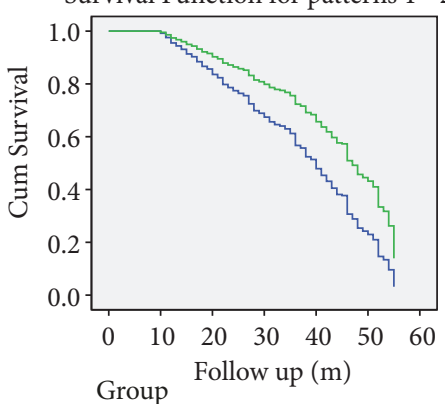

$\neg$ delayed PCI group

$\neg$ MT group

Survival Function for patterns 1 - 2

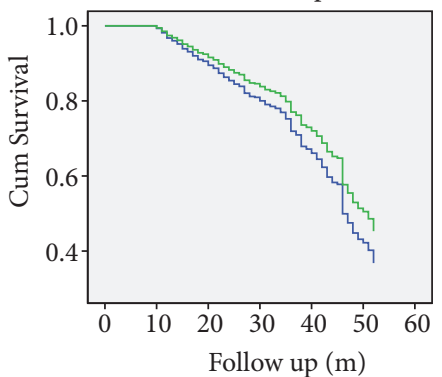

Group

$\neg$ delayed PCI group

$\neg$ MT group

Survival Function for patterns 1 - 2

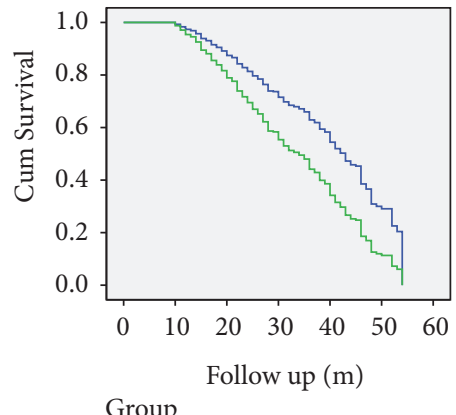

delayed PCI group

$\rightarrow$ MT group

Survival Function for patterns 1 - 2

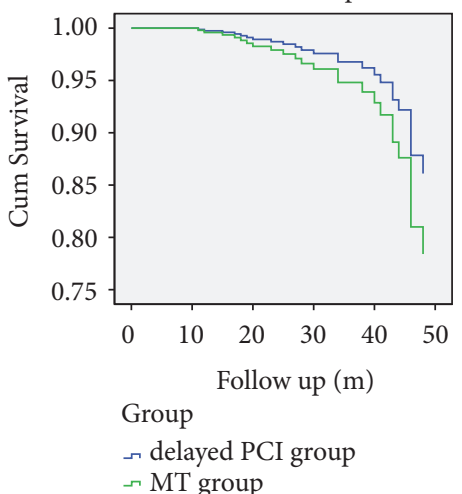

FIgure 1: Kaplan-Meier curves for event (ACM, CM, MACEs, Rehospitalization for HF, Revascularization, and Stroke, respectively) free survival according to therapy for STEMI after 12 hours of the onset of symptoms. (a) Before matching; (b) after matching. 
TABLE 3: Cox proportional hazards analysis of cardiac death, ACM, and MACEs.

\begin{tabular}{|c|c|c|}
\hline Characteristics & HR $(95 \%$ CI $)$ & P values \\
\hline \multicolumn{3}{|l|}{ Cardiac death } \\
\hline Age, yrs & $1.003(0.983-1.024)$ & 0.741 \\
\hline Gender & $1.477(0.840-2.596)$ & 0.175 \\
\hline LDL-C & $1.010(0.723-1.411)$ & 0.955 \\
\hline TC & $0.922(0.702-1.210)$ & 0.558 \\
\hline Current smoker & $1.345(0.779-2.321)$ & 0.288 \\
\hline Killip's class & $1.125(0.777-1.630)$ & 0.532 \\
\hline Treatment & $0.286(0.173-0.474)$ & $<0.001$ \\
\hline \multicolumn{3}{|l|}{$A C M$} \\
\hline Age, yrs & $1.007(0.988-1.025)$ & 0.489 \\
\hline Gender & $1.525(0.918-2.531)$ & 0.103 \\
\hline LDL-C & $1.086(0.824-1.431)$ & 0.559 \\
\hline TC & $0.902(0.712-1.143)$ & 0.304 \\
\hline Current smoker & $1.303(0.786-2.162)$ & 0.305 \\
\hline Killip’s class & $1.239(0.889-1.733)$ & 0.209 \\
\hline Treatment & $0.262(0.164-0.417)$ & $<0.001$ \\
\hline \multicolumn{3}{|l|}{ MACEs } \\
\hline Age, yrs & $0.995(0.985-1.009)$ & 0.319 \\
\hline Gender & $0.976(0690-1.379)$ & 0.889 \\
\hline LDL-C & $0.936(0.785-1.116)$ & 0.46 \\
\hline $\mathrm{TC}$ & $1.048(0.910-1.207)$ & 0.518 \\
\hline Current smoke & $1.011(0.786-1.301)$ & 0.93 \\
\hline Killip’s class & $0.987(0.793-1.229)$ & 0.91 \\
\hline Treatment & $0.723(0.563-0.929)$ & 0.011 \\
\hline
\end{tabular}

The TOSCA-2 trial [20], which was published in 2006, showed that the patency of IRA 1 year after PCI was much higher than that after medication therapy. However, the two groups showed no significant difference in LVEF $(\mathrm{P}=0.47)$. It was found that the long-term patency of the IRA being reopened in the recovery period of AMI was satisfactory. In 2008, Abbate A [21] conducted a meta-analysis of 10 randomized controlled trials, including the OAT study, the SWISSI II study, and the BRAVE-2 study, comparing the efficacy of PCI and medication therapy following STEMI (12 h-30 d). The results showed that mortality was significantly improved in the PCI group compared to that in the medication therapy group ( $\mathrm{OR}=0.49,95 \% \mathrm{CI}$ : $0.2-0.94, \mathrm{P}=0.03)$. PCI also outperformed medication therapy in cardiac remodeling $(\mathrm{P}=0.009)$. This meta-analysis showed that delayed reopening of the IRA ( $12 \mathrm{~h}-60 \mathrm{~d}$ ) could still improve cardiac function and survival. Degeare VS [22] believed that following AMI, IRA could prevent left ventricular remodeling, keeping the reopened vessels unobstructed and helping to achieve a TIMI grade 3 flow. Thus, PCI plays a crucial role in opening up the IRA and keeping it unobstructed, in preventing left ventricular remodeling and in protecting cardiac function. In our study, a multivariate Cox regression model found that delayed PCI was superior to simple medication therapy in terms of reducing cardiac death, all-cause death, and MACE $(\mathrm{HR}=0.286,95 \% \mathrm{CI}: 0.173-0.474, \mathrm{P}<0.001 ; \mathrm{HR}=0.262,95 \%$ CI: 0.164-0.417, $\mathrm{P}<0.001 ; \mathrm{HR}=0.723$, 95\% CI: 0.563-0.929, $\mathrm{P}=0.011$ ). Our results are consistent with the results of the meta-analysis observed by Abbate et al. [21]. Multivariate Cox regression analysis of the baseline clinical characteristics was performed to identify the influencing factors of cardiac death, all-cause death and MACE. The analysis showed that baseline clinical characteristics were not independent influencing factors for prognosis in STEMI patients. This result implied that there was potentially the presence of selection bias.

Our study was a retrospective trial. Delayed PCI was performed only for those surviving in the acute phase of AMI, and the critically ill patients who died in the acute phase were excluded. Patients receiving simple medication therapy were usually those who were older, had poor cardiac function and had underlying diseases. Therefore, patients receiving PCI tended to be those who were at a lower risk for complications compared with the patients receiving medication therapy. This was a major source of selection bias.

To reduce selection bias and potential confounding factors, PSM analysis was adopted for the adjustment of the baseline clinical characteristics. After 1:1 matching, 666 patients were selected for the final analysis, including 333 AMI patients who received delayed PCI and 333 AMI patients who received medication therapy.

Multivariate Cox regression analysis indicated that delayed PCI was superior to medication therapy in reducing both cardiac death and all-cause death $(\mathrm{HR}=0.221$. 95\% CI: 0.123-0.397, $\mathrm{P}<0.001 ; \mathrm{HR}=0.193,95 \% \mathrm{CI}: 0.112-0.334, \mathrm{P}<$ 0.001). This result is in agreement with the results from the meta-analysis conducted by Abbate A. Our research also 


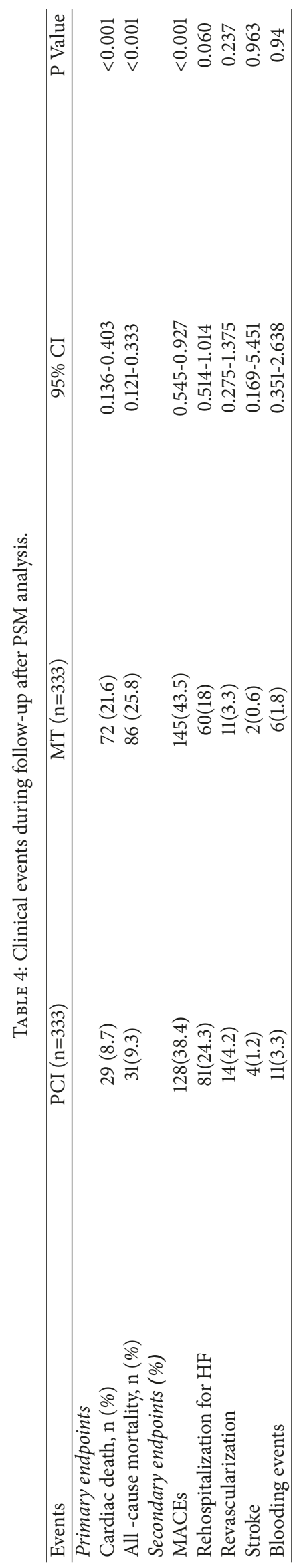


found that the incidence of MACE did not differ significantly between the delayed PCI group and medication therapy group $(\mathrm{P}=0.066)$. However, compared with medication therapy, delayed PCI greatly improved the prognosis of these patients $(12 \mathrm{~h}<\mathrm{t}<28 \mathrm{~d})$, and the risk of MACE was reduced in the delayed PCI group more so than in the medication therapy group (HR=0.766, 95\% CI: 0.566-1.018). Multivariate Cox regression analysis was performed on the baseline clinical characteristics to assess the influencing factors of cardiac death, all-cause death, and MACE. Killip grade was an independent influencing factor of cardiac death and allcause death in STEMI patients. This result may be related to the rematching of patients and to the selection of the baseline clinical characteristics.

Time is the most important factor in the reopening of IRA. However, none of the patients in our study received early reperfusion therapy. The reasons for this may include the following: (1) delayed diagnosis: the patients were usually not fully aware of the danger of AMI, and less specialized hospitals lacked experience in making an early diagnosis of AMI; (2) fear of the risk that PCI posed in elderly patients: many elderly patients did not receive PCI because of their advanced age, which reflects concern with the safety of PCI the physicians. In fact, for elderly AMI patients, the risk of death is high with or without PCI; (3) the patients and their relatives were hesitant in making a treatment decision, which led to a delayed intervention.

It will be of a high significance to assess the benefits of late reperfusion for AMI beyond a $12 \mathrm{~h}$ window but not exceeding 2-60 d. 2017 ESC STEMI guidelines give a class IIaB recommendation for primary PCI (PPCI) beyond $12 \mathrm{hrs}$ [22]. 2018 myocardial revascularization ESC/EACTS guidelines give a class $\mathrm{IIaB}$ recommendation for a routine primary PCI strategy that indicates that primary PCI should be considered in patients presenting late $(12-48 \mathrm{~h})$ after the onset of symptoms [23]. However, selective PCI cannot salvage the dead cardiomyocytes in the acute phase of AMI but can rather improve cardiac function and prognosis by reducing myocardial remodeling and promoting functional recovery of the surviving myocardium in the infarct region. Selective PCI may improve prognosis through several pathways: (1) restoring antegrade flow in the vessels supplying the infarction region, thus salvaging the hibernating myocardium, and alleviating reversible myocardial damage and promoting its functional recovery; (2) improving scar repair of the infarct tissues, reducing myocardial remodeling in the noninfarct region, and preventing the extension of infarct region and ventricular dilation; (3) reducing electrical instability of the myocardium and malignant ventricular arrhythmia; (4) increasing collateral circulation near the infarct and alleviating myocardial ischemia.

\section{Limitations}

Our study has the following limitations. First, for a retrospective study design, the research findings were closely related to the accuracy and integrity of medical records. Second, the patients were not selected by random sampling but were all patients at the department of cardiology at the First Affiliated
Hospital of Xinjiang Medical University, which may give rise to selection bias. Finally, several variables, such as the interval from the onset of symptoms to diagnosis and the ejection fraction, were not included in the PSM analysis. Furthermore, there may be some residual unknown confounding factors in our analysis despite PSM being performed.

\section{Conclusions}

PCI delayed beyond a $12 \mathrm{~h}$ window for STEMI patients was conducive to reducing long-term mortality.

\section{Abbreviations \\ PPCI: Primary percutaneous coronary intervention \\ STEMI: ST-segment elevation myocardial infarction \\ PSM: Propensity score matching \\ AMI: Acute myocardial infarction \\ CAG: Coronary angiography \\ LVEF: Left ventricular ejection fraction \\ IRA: Infarct-related artery \\ ACM: All-cause mortality \\ MACE: Major adverse cardiovascular events.}

\section{Data Availability}

Due to confidentiality policies, the data used in this study will not be shared.

\section{Ethical Approval}

The research protocol for this study was approved by the ethics committee or review committee of the First Affiliated Hospital of Xinjiang Medical University.

\section{Consent}

Because the study was a retrospective cohort study based on real-world situations, there was no need to obtain informed consent from the patients.

\section{Disclosure}

The abstract of this study has been published in the Journal of the American College of Cardiology (https://doi.org/10.1016/ j.jacc.2018.08.609). None of the funding sources played a role in the design of the study, in the collection, analysis or interpretation of the data, or the decision to submit the manuscript for publication.

\section{Conflicts of Interest}

No potential conflicts of interest relevant to this article were reported by any of the authors.

\section{Authors' Contributions}

Wen-Juan Xiu and Hai-Tao Yang made substantial contributions to the study conception and design and the drafting and 
critical revision of the manuscript for important intellectual content. Ying-Ying Zheng made substantial contributions to the study conception and design and to the critical revision of the manuscript for important intellectual content. Yi-Tong $\mathrm{Ma}$ and Xiang Xie made substantial contributions to the study conception and design and to the drafting and critical revision of the manuscript for important intellectual content, as well as the supervision of the study.

\section{References}

[1] C. M. Gibson, Y. B. Pride, P. D. Frederick et al., "Trends in reperfusion strategies, door-to-needle and door-to-balloon times, and in-hospital mortality among patients with ST-segment elevation myocardial infarction enrolled in the National Registry of Myocardial Infarction from 1990 to 2006," American Heart Journal, vol. 156, no. 6, pp. 1035-1044, 2008.

[2] K. A. Eagle, B. K. Nallamothu, R. H. Mehta et al., "Trends in acute reperfusion therapy for ST-segment elevation myocardial infarction from 1999 to 2006: We are getting better but we have got a long way to go," European Heart Journal, vol. 29, no. 5, pp. 609-617, 2008.

[3] Cardiology Branch of Chinese Medical Association, "Editorial Board of Zhong Hua Xin Xue Bing Xue Za Zhi. Guidelines for the diagnosis and treatment of acute ST - segment elevation myocardial infarction," Zhong Hua Xin Xue Guan Bing Xue Za Zhi, vol. 43, pp. 380-393, 2015.

[4] G. N. Levine, E. R. Bates, J. C. Blankenship et al., “2015 ACC/AHA/SCAI focused update on primary percutaneous coronary intervention for patients with st-elevation myocardial infarction: an update of the 2011 ACCF/AHA/SCAI guideline for percutaneous coronary intervention and the 2013 ACCF/AHA guideline for the management of st-elevation myocardial infarction: a report of the american college of cardiology/american heart association task force on clinical practice guidelines and the society for cardiovascular angiography and interventions," in Circulation, vol. 133, pp. 1315-1147, 2016.

[5] P. T. O'Gara, F. G. Kushner, D. D. Ascheim et al., “2013 ACCF/AHA guideline for the management of st-elevation myocardial infarction: a report of the American College of Cardiology Foundation/American Heart Association Task Force on Practice Guidelines," Journal of the American College of Cardiology, vol. 61, no. 4, pp. e78-e140, 2013.

[6] F. Van de Werf, J. Bax, A. Betriu et al., "Management of acute myocardial infarction in patients presenting with persistent stsegment elevation: the task force on the management of stsegment elevation acute myocardial infarction of the european society of cardiology," European Heart Journal, vol. 29, pp. 2909-2945, 2008.

[7] M. Roffi, C. Patrono, J.-P. Collet et al., "Management of acute coronary syndromes in patients presenting without persistent st-segment elevation of the european society of cardiology. 2015 esc guidelines for the management of acute coronary syndromes in patients presenting without persistent st-segment elevation: task force for the management of acute coronary syndromes in patients presenting without persistent st-segment elevation of the european society of cardiology (esc)," European Heart Journal, vol. 37, pp. 267-315, 2016.

[8] H. M. Yuan, W. U. Qh, N. X. Wen et al., "Effect of intervention and drug therapy on left ventricular function in patients with acute myocardial infarction without segment elevation," Wu Jing Hou Qin Xue Yuan Xue Bao, vol. 23, pp. 471-473, 2014.
[9] X. Fu and J. Lu, "Different statins in the treatment of patients with early-stage coronary heart disease acute myocardial infarction," Zhong Guo Yi Yao Zhi Nan, vol. 3, pp. 5-6, 2015.

[10] X.-L. Wu, R. Zhu, H. Jiang, and B. Li, "Different treatment interventions affect plasma NT-ProBNP levels and early exercise tolerance in patients with acute ST-segment elevation myocardial infarction," Postgraduate Medical Journal, vol. 124, no. 2, pp. 58-63, 2012.

[11] U. Zeymer, R. Uebis, A. Vogt et al., "Randomized comparison of percutaneous transluminal coronary angioplasty and medical therapy in stable survivors of acute myocardial infarction with single vessel disease: a study of the Arbeitsgemeinschaft Leitende Kardiologische Krankenhausärzte," Circulation, vol. 108, no. 11, pp. 1324-1328, 2003.

[12] P. Erne, A. W. Schoenenberger, D. Burckhardt et al., "Effects of percutaneous coronary interventions in silent ischemia after myocardial infarction: The SWISSI II randomized controlled trial," Journal of the American Medical Association, vol. 297, no. 18, pp. 1985-1991, 2007.

[13] W. Xu, D. N. Holmes, R. C. Becker, M. T. Roe, E. D. Peterson, and T. Y. Wang, "Comparison of long-term outcomes between older Asian and white patients with non-ST-segment elevation myocardial infarction: Findings from CRUSADE-CMS database," American Heart Journal, vol. 166, no. 6, pp. 1050-1055, 2013.

[14] L. J. Gao and J. L. Chen, "Advances in treatment of acute coronary syndromes," China Journal of Circulation, vol. 28, pp. 3-5, 2013.

[15] Y. H. Fu, D. W Suo, and F. Peng, "Study on delayed emergency PCI in the treatment of acute ST-segment elevation myocardial infarction and prognosis of patients," Chongqing Yi Xue, vol. 33, pp. 4479-4480, 2014.

[16] A. Abbate, G. G. L. Biondi-Zoccai, D. L. Appleton et al., "Survival and cardiac remodeling benefits in patients undergoing late percutaneous coronary intervention of the infarct-related artery: evidence from a meta-analysis of randomized controlled trials," Journal of the American College of Cardiology, vol. 51, no. 9, pp. 956-964, 2008.

[17] J. S. Hochman, G. A. Lamas, C. E. Buller et al., "Coronary intervention for persistent occlusion after myocardial infarction," The New England Journal of Medicine, vol. 355, no. 23, pp. 23952407, 2006.

[18] V. Džavík, C. E. Buller, G. A. Lamas et al., "Randomized trial of percutaneous coronary intervention for subacute infarctrelated coronary artery occlusion to achieve long-term patency and improve ventricular function: The Total Occlusion Study of Canada (TOSCA)-2 trial," Circulation, vol. 114, no. 23, pp. 24492457, 2006

[19] Cardiology Branch of Chinese Medical Association, "Editorial Board of Zhong Hua Xin Xue Bing Xue Za Zhi," in Guidelines for Percutaneous Coronary Intervention in China, vol. 44, pp. 382400, 2016.

[20] A. Abbate, G. G. Biondi-Zoccai, D. L. Appleton et al., "Survival and cardiac remodeling benefits in patients undergoing late percutaneous coronary intervention of the infarct-related artery: evidence from a meta-analysis of randomized controlled trials," Journal of the American College of Cardiology, vol. 51, no. 9, pp. 956-964, 2008.

[21] V. S. DeGeare, G. Dangas, G. W. Stone, and C. L. Grines, "Interventional procedures in acute myocardial infarction," American Heart Journal, vol. 141, no. 1, pp. 15-24, 2001. 
[22] B. Ibanez, S. James, S. Agewall et al., "ESC Scientific Document Group. 2017 ESC Guidelines for the management of acute myocardial infarction in patients presenting with ST-segment elevation: The Task Force for the management of acute myocardial infarction in patients presenting with ST-segment elevation of the European Society of Cardiology (ESC)," European Heart Journal, vol. 39, no. 2, pp. 119-177, 2018.

[23] F. J. Neumann, M. Sousa-Uva, A. Ahlsson et al., "ESC Scientific Document Group. 2018 ESC/EACTS Guidelines on myocardial revascularization," European Heart Journal, vol. 40, no. 2, pp. 87-165, 2019. 


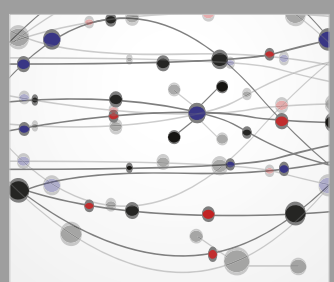

The Scientific World Journal
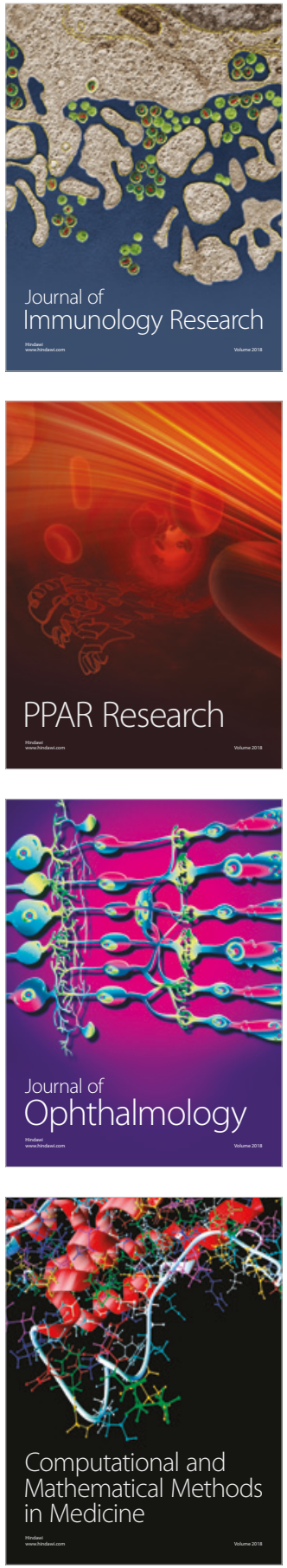

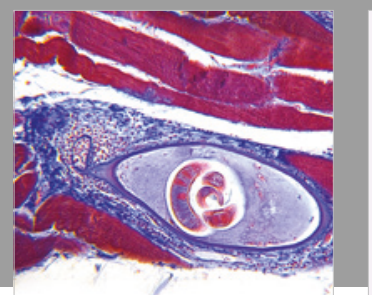

Gastroenterology Research and Practice

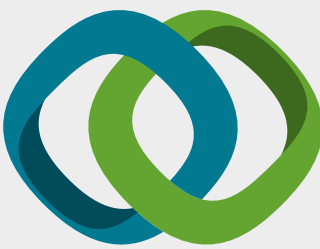

\section{Hindawi}

Submit your manuscripts at

www.hindawi.com
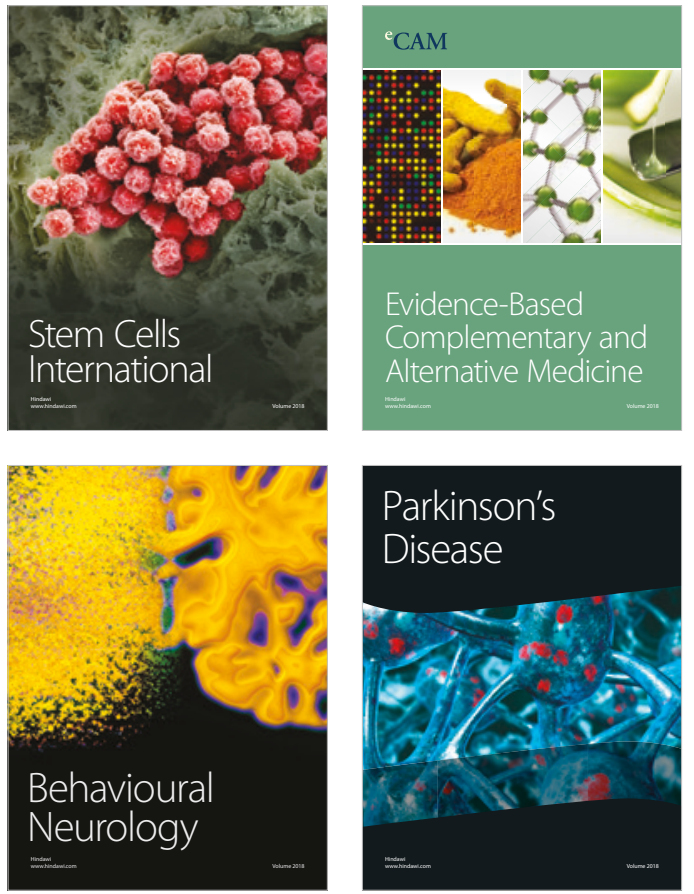

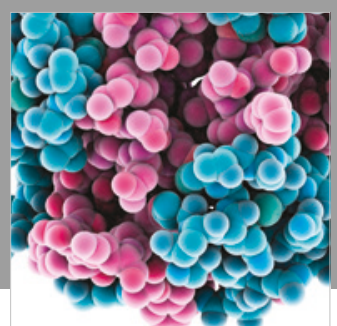

ournal of

Diabetes Research

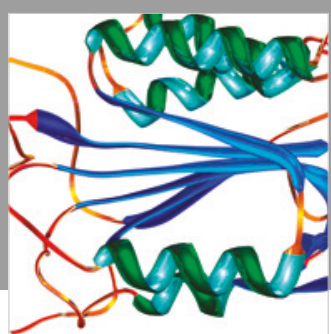

Disease Markers
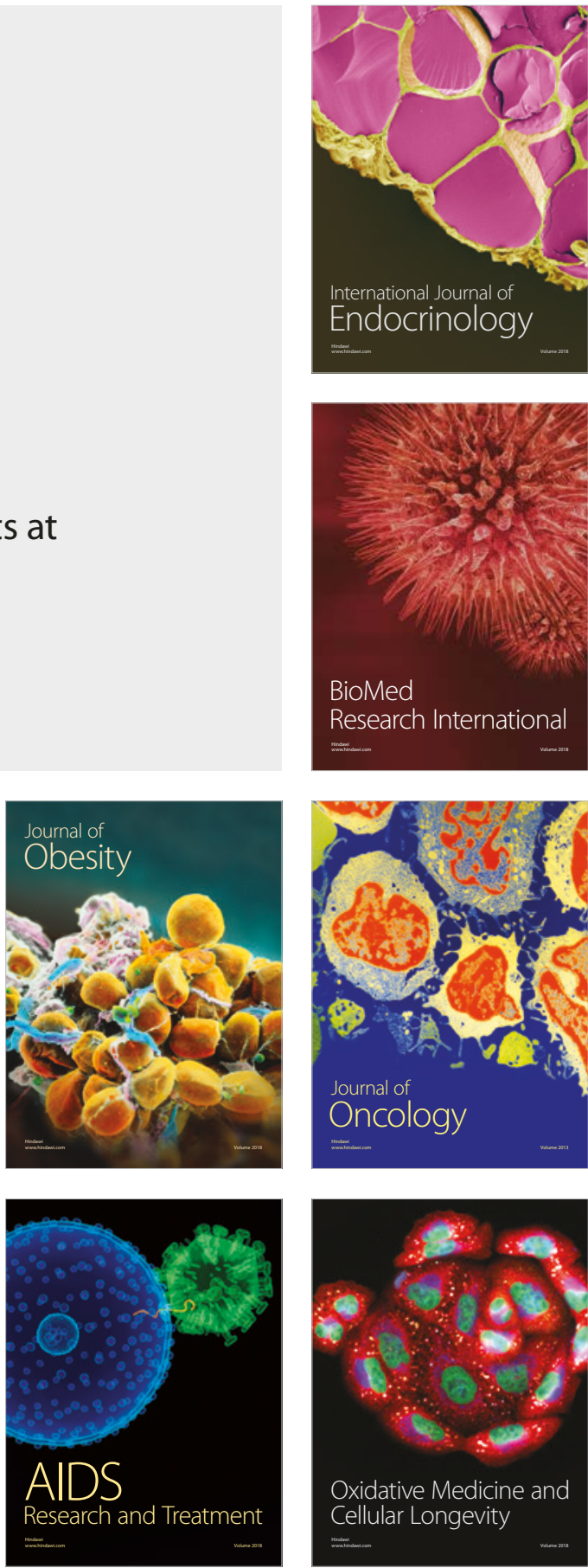